\title{
Frecuencia de micobacterias ambientales en Chile en el año 2008
}

\begin{abstract}
ANGÉLICA SCAPPATICCIO B.*, MARITZA VELASCO R.*, TAMARA LEIVA C.* y JUAN CARLOS RODRÍGUEZ D.**
\end{abstract}

\section{Frequency of environmental mycobacterium in Chile. 2008}

Objective: To know the frequency of environmental mycobacterium isolations in Chile in the year 2008. Methods: 600 AFB (acid fast bacilli) positive cultures from 22 laboratories of Tuberculosis Bacteriology of the different Network Health Services that constitute the Tuberculosis Control Program of the country were studied, during four months at 2008. 545 (90.8\%) were pulmonary and 55 (9.2\%) extra pulmonary. Acid fast bacilli smears were confirmed by Ziehl Neelsen and identification of mycobacteria species or complex were identified by traditional tests according to Runyon classification and biochemical tests, genetic probes and pattern analysis restriction (PRA). Results: 585 cultures were appropriated for inclusion in the study. In 91.3\% $(n=534)$ of the cases Mycobacterium tuberculosis was isolated while $0.3 \%$ was Mycobacterium bovis subspecie BCG $(n=3)$ and $8.4 \%(n=48)$ corresponded to environmental mycobacterium. Of the latter, Mycobacterium kansasii (2.6\%), Mycobacterium avium-intracellulare (1.5\%) and Mycobacterium chelonae (1.0\%) were the most commonly isolated. Conclusion: According to the figures of this study and comparing them with studies of previous years (1988 and 1998) it is concluded that the number of environmental mycobacterium isolated has been relatively constant during the last decade, as well as the species, more commonly isolated.

Key words: Environmental mycobacterium, non tuberculosis mycobacterium, atypical mycobacterium.

\section{Resumen}

Objetivos: Conocer la frecuencia de aislamientos de micobacterias ambientales en Chile en el año 2008. Material y Métodos: Se recibieron 600 cultivos desde 22 laboratorios de Bacteriología de la Tuberculosis de los distintos Servicios de Salud de la red que comprende el Programa de Control de la Tuberculosis del país, durante un período de cuatro meses del año 2008. Quinientos cuarenta y cinco (90,8\%) correspondieron a localización pulmonar y 55 (9,2\%) a extrapulmonar. Se confirmó la alcohol-ácido resistencia por tinción de Ziehl Neelsen y para la identificación de especies o complejos micobacterianos se utilizaron pruebas tradicionales y bioquímicas de acuerdo al criterio de clasificación de Runyon, sondas genéticas y análisis de patrones de restricción (PRA). Resultados: De los 600 cultivos recibidos, 585 fueron aptos para ser incluidos en el estudio. De éstos, en el 91,3\% ( $n=534)$ de los casos se aisló Mycobacterium tuberculosis, en un 0,3\% Mycobacterium bovis subespecie BCG (n $=3)$ y un 8,4\% $(n=48)$ correspondió a micobacterias ambientales. De estas últimas, Mycobacterium kansasii (2,6\%), Mycobacterium avium-intracellulare (1,5\%) y Mycobacterium chelonae (1,0\%) fueron las más comúnmente aisladas. Conclusiones: De acuerdo a las cifras obtenidas en este estudio y comparadas con estudios de años anteriores (1988 y 1998) se concluye que el número de aislamientos de micobacterias ambientales permaneció relativamente constante esta última década, como también las especies más comúnmente aisladas.

Palabras clave: Micobacterias ambientales, micobacterias no tuberculosas, micobacterias atípicas.

\footnotetext{
* Sección Micobacterias, Laboratorio Biomédico Nacional, Instituto de Salud Pública de Chile.

** Instituto Nacional del Tórax.

Financiamiento: Instituto de Salud Pública.
} 


\section{Introducción}

Las micobacterias ambientales (también llamadas micobacterias atípicas, micobacterias no tuberculosas o micobacterias distintas de $\mathrm{Myco-}$ bacterium tuberculosis) $)^{1,2}$ son un grupo fascinante de patógenos de mamíferos, de aves y oportunistas humanos, que pueden provocar morbilidad y mortalidad humana e impactos económicos importantes en la agricultura ${ }^{3}$.

Las micobacterias ambientales (MA) fueron descritas poco tiempo después de que Koch descubrió el bacilo tuberculoso el año 1882. Se denominaron micobacterias atípicas para diferenciarlas del Mycobacterium tuberculosis; inicialmente fueron consideradas como contaminantes ambientales o simples colonizadoras, aunque patógenas para mamíferos y aves, A partir de 1950 se reconoció su capacidad de provocar también morbi-mortalidad en seres humanos ${ }^{4}$.

Las MA se distinguen de los miembros del complejo M. tuberculosis por el hecho de no ser patógenos obligados y por ser habitantes del medio ambiente, en una gran variedad de reservorios como agua natural y potable, suelo, aerosoles, protozoos, animales, incluyendo peces, aves y seres humanos, y porque pueden ser encontrados como saprófitos, comensales y simbióticos ${ }^{2-8}$. Su modo de transmisión no está bien definido, pero la exposición al medio ambiente parece ser el principal factor ${ }^{2,9}$. Se adquieren por inhalación o ingestión; la trasmisión persona a persona es muy poco frecuente y su patogenicidad está relacionada con las condiciones inmunológicas del huésped ${ }^{2,4}$.

Son considerados como agentes oportunistas causantes de infecciones pulmonares y extrapulmonares, especialmente en inmunocomprometidos, pero también en individuos inmunocompetentes portadores de patología pulmonar crónica $^{2,4,10-16}$. Presentan resistencia a la mayoría de los agentes antituberculosos ${ }^{2}$ y producen enfermedades que se conocen con el nombre genérico de micobacteriosis, que aunque de baja incidencia, aumentan su frecuencia relativa a medida que disminuye la tuberculosis propiamente tal en una comunidad². También se ha confirmado su presencia como contaminantes en aerosoles de agua caliente y en inyectables lipolíticos utilizados en tratamientos con fines estéticos ${ }^{17}$.

Las enfermedades producidas por MA han tenido un importante incremento en los últimos años en todo el mundo $2,4,8,10,12,13,14$. Las razones de este aumento son múltiples: menor prevalencia de la infección por $M$. tuberculosis, mejor disponibilidad de técnicas de laboratorio para aislarlas e identificarlas, aumento de la población inmunocomprometida VIH/SIDA y aumento en la sobrevida de individuos con enfermedades debilitantes como cáncer, diabetes, trasplantados, uso de corticoides y enfermedades autoinmunes y pulmonares crónicas ${ }^{2-4}$.

El aumento de los aislamientos de MA, también ha sido observado en nuestro país. En 1967, en el Laboratorio Nacional de Referencia del Instituto de Salud Pública de Chile se creó el Laboratorio de Identificación de Micobacterias del Centro de Referencia de Microbiología de la Tuberculosis, implementándose las técnicas clásicas de identificación de Micobacterias. Esto permitió elaborar las "Normas de Tipificación"18 en base a la experiencia del trabajo de este laboratorio y a los estudios de frecuencia de MA efectuados en el país ${ }^{18-24}$, vigentes hasta hoy (Normas MINSAL 2005). En 1970 se efectuó nuestra primera publicación ${ }^{20}$ y desde entonces ha existido la preocupación constante de mantener cifras actualizadas de la frecuencia con que ocurren estos aislamientos en los laboratorios de la red y la importancia clínica de éstos ${ }^{18}$.

El objetivo de este estudio es conocer la frecuencia de aislamientos de MA en Chile durante 2008.

\section{Materiales y Métodos}

Cepas. Se recibieron 600 cultivos procedentes de laboratorios de bacteriología de la tuberculosis de 22 hospitales de la red que conforman el Programa de Control de la Tuberculosis del país, desarrollados en medio Lowenstein-Jensen y con tinción de Ziehl Neelsen positiva de las colonias.

$\mathrm{El}$ antecedente de tratamiento fue consignado en cada caso.

El estudio se realizó con muestras obtenidas en el período comprendido entre el 1 de julio y el 30 de octubre de 2008.

Identificación fenotípica. Se registró la procedencia, el tipo de muestra, antecedente de co-infección con VIH, número de colonias y características macroscópicas. Se realizó un subcultivo de cada cultivo, el que fue observado a los 4, 14, 21, 28 y 60 días. Se estudió la capacidad de crecimiento a 25,37 y $45{ }^{\circ} \mathrm{C}$, formación de pigmentos, producción de niacina, pruebas de actividad enzimática como nitrato reductasa, catalasa, hidrólisis del tween, ureasa y tolerancia a $\mathrm{NaCl}$ al $5 \%$.

En caso de existir sospecha de $M$. bovis o $M$. bovis sub-especie BCG se realizó prueba de cre- 
cimiento en presencia de TCH (thiophene carboxylic acid hydrazide), estudio de susceptibilidad a cicloserina y presencia de pirazinamidasa.

$\boldsymbol{P R A}$. Las cepas también fueron analizadas mediante análisis de patrones de restricción (PRA) según Araya et $\mathrm{al}^{25}$.

Sondas genéticas. Los cultivos con características morfológicas especiales fueron analizados por sondas genéticas específicas para el complejo $M$. tuberculosis, $M$. kansasii, M. gordonae y complejo MAI (Accuprobe).

\section{Resultados}

Se recibieron 600 cultivos de 22 hospitales de la red de tuberculosis. De éstos, 2 no crecieron, 7 se contaminaron en el sub-cultivo y en 6 crecieron colonias del género Nocardia, que no fueron consideradas para el análisis, dando un total de 585 cepas en estudio.

En un 91,3\% (534) de los cultivos se aisló complejo Mycobacterium tuberculosis, en un 0,3\% Mycobacterium bovis subespecie BCG y en el 8,4 \% (51) restante MA (Tabla 1).

De las MA aisladas, un 31,3\% (15) correspondió a M. kansasii, 18,8\% (9) a M. avium (complejo MAI), $12,5 \%$ (6) a $M$. chelonae y $10,4 \%$ (5) a $M$. fortuitum y M. gordonae (Tabla 2), como las especies más comúnmente aisladas.

Se relacionó la frecuencia de los aislamientos de Mycobacterium tuberculosis y de MA con el tipo de muestra (Tabla 3). En muestras pulmonares se aisló un 91,7\% de $M$. tuberculosis (489) $\mathrm{y}$ un $8,3 \%$ (44) de MA, mientras en muestras extra-pulmonares se aislaron 86,5\% (45) de $M$. tuberculosis, 5,8\% (3) de $M$. bovis subespecie BCG y un 7,7\% (4) de MA. Los porcentajes más altos de estas últimas se obtuvieron en muestras de sangre, lavado broncoalveolar y orina, y de $\mathrm{M}$. bovis BCG de pus vertebral y ganglionar.

La Tabla 4 resume la frecuencia de las especies de micobacterias aisladas en cada tipo de muestra. Se observa que en todas ellas predomina $M$. tuberculosis excepto en sangre, donde lo hace la especie $M$. avium, y en pus vertebral, donde se encuentra M. tuberculosis y M. bovis subespecie $B C G$ en igual proporción.

En la Tabla 5 se muestra el aislamiento de las micobacterias según el tipo de muestra en pacientes VIH/SIDA y en la Tabla 6 las cepas aisladas más comúnmente en estos pacientes: $M$. tuberculosis, seguido de M. kansasii y M. avium.

En la Tabla 7 se relaciona el tipo de micobac-
Tabla 1. Frecuencia de aislamientos de micobacterias

\begin{tabular}{|lrr|}
\hline Especie & n & \% \\
\hline M. tuberculosis & 534 & 91,3 \\
M. bovis subespecie BCG & 3 & 0,5 \\
M. kansasii & 15 & 2,6 \\
M. avium-intracellulare & 9 & 1,5 \\
M. chelonae & 6 & 1,0 \\
M. fortuitum & 5 & 0,9 \\
M. gordonae & 5 & 0,9 \\
M. peregrinum & 3 & 0,5 \\
C. M. terrae & 2 & 0,3 \\
M. abscessus & 1 & 0,2 \\
Complejo M. fortuitum chelonae & 1 & 0,2 \\
MNT de crecimiento rápido, & 1 & 0,2 \\
cromógena & & \\
\hline Total & 585 & 100 \\
\hline
\end{tabular}

MNT = micobacteria no tuberculosis.

Tabla 2. Frecuencia de micobacterias ambientales

\begin{tabular}{|lcc|}
\hline Especie & n & \% \\
\hline M. kansasii & 15 & 31,3 \\
\hline M. avium-intracellulare (MAI) & 9 & 18,8 \\
\hline M. chelonae & 6 & 12,5 \\
\hline M. fortuitum & 5 & 10,4 \\
\hline M. gordonae & 5 & 10,4 \\
M. peregrinum & 3 & 6,3 \\
\hline CM terrae & 2 & 4,2 \\
\hline M. abscessus & 1 & 2,1 \\
\hline Complejo M. fortuitum chelonae & 1 & 2,1 \\
\hline $\begin{array}{l}\text { MNT de crecimiento rápido, } \\
\text { cromógena }\end{array}$ & 1 & 2,1 \\
Total & 48 & 100 \\
\hline
\end{tabular}

MNT = micobacteria no tuberculosis.

teria aislada con el antecedente de tratamiento antituberculoso, en la cual podemos observar que la mayor proporción de MA aparece en muestras de pacientes con antecedentes de tratamientos previos.

\section{Discusión}

Este estudio se realizó con 585 muestras de 44.136 cultivos realizados en el período por los Servicios de Salud participantes, lo cual representa un $27 \%$ de los cultivos efectuados en el país el 
Tabla 3. Frecuencia de micobacterias según el tipo de muestra

\begin{tabular}{|c|c|c|c|c|c|c|c|}
\hline \multirow[t]{2}{*}{ Tipo de muestra } & \multicolumn{2}{|c|}{ M. tuberculosis } & \multicolumn{2}{|c|}{ M. bovis BCG } & \multicolumn{2}{|c|}{ MA* } & \multirow[t]{2}{*}{ Total } \\
\hline & Total & $\%$ & Total & $\%$ & Total & $\%$ & \\
\hline Pulmonar & 489 & 91,7 & 0 & 0 & 44 & 8,3 & 533 \\
\hline Extrapulmonar & 45 & 86,5 & 3 & 5,8 & 4 & 7,7 & 52 \\
\hline Total & 534 & 91,3 & 3 & 0,5 & 48 & 8,2 & 585 \\
\hline Expectoración & 472 & 91,8 & 0 & 0 & 42 & 8,2 & 514 \\
\hline Contenido gástrico & 4 & 100 & 0 & 0 & 0 & 0 & 4 \\
\hline LBA & 13 & 86,7 & 0 & 0 & 2 & 13,3 & 15 \\
\hline Líquido pleural & 6 & 100 & 0 & 0 & 0 & 0 & 6 \\
\hline Ganglionar & 6 & 75 & 2 & 100 & 0 & 0 & 8 \\
\hline Tejido & 5 & 100 & 0 & 0 & 0 & 0 & 5 \\
\hline Absceso & 6 & 100 & 0 & 0 & 0 & 0 & 6 \\
\hline LCR & 5 & 100 & 0 & 0 & 0 & 0 & 5 \\
\hline Sangre & 2 & 40 & 0 & 0 & 3 & 60 & 5 \\
\hline Biopsia vesical & 1 & 100 & 0 & 0 & 0 & 0 & 1 \\
\hline Orina & 11 & 91,7 & 0 & 0 & 1 & 8,3 & 12 \\
\hline Pus vertebral & 1 & 50,0 & 1 & 50 & 0 & 0 & 2 \\
\hline Muestra no especificada & 2 & 100 & 0 & 0 & 0 & 0 & 2 \\
\hline Total & 534 & 91,3 & 3 & 0,5 & 48 & 8,2 & 585 \\
\hline
\end{tabular}

LBA: lavado broncoalveolar; LCR: líquido cefalorraquídeo. *Micobacterias ambientales.

año 2008 (161.944), lo que valida los resultados obtenidos.

El aumento de las afecciones por MA es un fenómeno conocido y descrito mundialmente. Este incremento ha sido relativamente paulatino en el tiempo en nuestro medio (Tabla 8) y el resultado actual demuestra un aumento en $2,6 \%$ en muestras pulmonares y $2,9 \%$ en muestras extrapulmonares, respecto a los resultados obtenidos en décadas anteriores en el país, con hallazgos de $5,7 \%$ en muestras pulmonares y 4,8\% extrapulmonares (Velasco M., ISP de Chile 1998-1999). También concuerdan estos resultados con las especies más comúnmente aisladas en otros países como España, Cuba y México ${ }^{26-28}$.

La evaluación de la frecuencia de aislamientos de Mycobacterium tuberculosis y MA en relación al tipo de muestra, mostró que los mayores porcentajes de aislamientos de MA correspondieron a sangre, y los de Mycobacterium bovis BCG a ganglios. Aunque el número de aislamientos en este tipo de muestras fueron escasos, los resultados concuerdan con los de la literatura y con la experiencia práctica, lo que permite afirmar que efectivamente, en sangre, Mycobacterium avium, $\mathrm{y}$ en muestras ganglionares Mycobacterium bovis
BCG, corresponderían a las especies no TB más comúnmente aisladas. En las muestras ganglionares con Mycobacterium bovis BCG, estas se encontraron en niños menores de un año, lo que obviamente hace sospechar de una complicación en la aplicación de la vacuna BCG, que sólo correspondieron a linfoadenitis y no a infecciones generalizadas o BCGitis.

A pesar del escaso número de aislamientos, sangre, orina y lavado bronco-alveolar son los tipos de muestras que más presentan MA, concordando con la literatura y con estudios anteriores. Es sabido que en el caso de la orina y contenido gástrico, por su naturaleza mixta, vale decir su gran interrelación con la naturaleza y por lo tanto con los nichos ecológicos de las MA, éstas podrían aislarse con más frecuencia sin ser patógenas. Sin embargo, sólo fueron encontradas con más frecuencia en orina, no así en contenido gástrico, donde sólo se encontró Mycobacterium tuberculosis. En sangre esta mayoría es a expensas de Mycobacterium avium, como lo demostraron trabajos anteriores e internacionales ${ }^{26,29}$. En el caso de lavado bronco-alveolar, trabajos anteriores mostraron que las técnicas de toma de muestra pueden contribuir a contaminarlas dando 
Tabla 4. Frecuencia de micobacterias ambientales según tipo de muestras

\begin{tabular}{|c|c|c|c|}
\hline $\begin{array}{l}\text { Tipo de muestra } \\
\text { (sitio de la lesión) }\end{array}$ & Especie & $\mathbf{n}$ & $\%$ \\
\hline Expectoración & $\begin{array}{l}\text { M. tuberculosis } \\
\text { M. kansassi } \\
\text { M. chelonae } \\
\text { MAI } \\
\text { M. fortuitum } \\
\text { M. gordonae } \\
\text { M. peregrinum } \\
\text { M. terrae } \\
\text { M. abscessus } \\
\text { Complejo fortuitum-chelonae } \\
\text { M. grupo IV- cromógena } \\
\text { MA* }\end{array}$ & $\begin{array}{r}472 \\
14 \\
6 \\
5 \\
5 \\
4 \\
3 \\
2 \\
1 \\
1 \\
1 \\
42\end{array}$ & $\begin{array}{r}91,8 \\
2,7 \\
1,2 \\
1 \\
1 \\
0,8 \\
0,6 \\
0,4 \\
0,2 \\
0,2 \\
0,2 \\
8,2\end{array}$ \\
\hline Contenido gástrico & M. tuberculosis & 4 & 100 \\
\hline Lavado bronco-alveolar & $\begin{array}{l}\text { M. tuberculosis } \\
\text { M. kansasii } \\
\text { MAI } \\
\text { MA* }\end{array}$ & $\begin{array}{r}13 \\
1 \\
1 \\
2\end{array}$ & $\begin{array}{r}86,7 \\
6,7 \\
6,7 \\
13,3\end{array}$ \\
\hline Líquido pleural & M. tuberculosis & 6 & 100 \\
\hline Ganglionar & $\begin{array}{l}\text { M. tuberculosis } \\
\text { M. bovis BCG }\end{array}$ & $\begin{array}{l}6 \\
2\end{array}$ & $\begin{array}{l}75 \\
25\end{array}$ \\
\hline Muestra de tejidos & M. tuberculosis & 5 & 100 \\
\hline Absceso & M. tuberculosis & 6 & 100 \\
\hline LCR & M. tuberculosis & 5 & 100 \\
\hline Sangre & $\begin{array}{l}\text { M. tuberculosis } \\
\text { MA* }\end{array}$ & $\begin{array}{l}2 \\
3\end{array}$ & $\begin{array}{l}40 \\
60\end{array}$ \\
\hline Biopsia vesical & M. tuberculosis & 1 & 100 \\
\hline Orina & $\begin{array}{l}\text { M. tuberculosis } \\
\text { MA* }\end{array}$ & $\begin{array}{r}11 \\
1\end{array}$ & $\begin{array}{r}91,7 \\
8,3\end{array}$ \\
\hline Pus vertebral & $\begin{array}{l}\text { M. bovis BCG } \\
\text { M. tuberculosis }\end{array}$ & $\begin{array}{l}1 \\
1\end{array}$ & $\begin{array}{l}50 \\
50\end{array}$ \\
\hline Muestra no especificada & M. tuberculosis & 2 & 100 \\
\hline Total & & 585 & \\
\hline
\end{tabular}

*MA: Micobacterias ambientales; LCR: líquido céfalorraquídeo.

Tabla 5. Aislamientos en personas con infección VIH/SIDA según tipo de muestra

\begin{tabular}{|llrr|}
\hline Tipo de muestra & Especies aisladas & n & \% \\
\hline Expectoración & M. tuberculosis & 15 & 57,7 \\
& M. kansasii & 3 & 11,5 \\
M. chelonae & 1 & 3,8 \\
\hline \multirow{2}{*}{ Sangre } & M. tuberculosis & 2 & 7,7 \\
& MAI & 2 & 7,7 \\
Ganglionar & M. tuberculosis & 1 & 3,8 \\
\hline Lavado bronco-alveolar & M. tuberculosis & 1 & 3,8 \\
Total & & 1 & 3,8 \\
\hline
\end{tabular}

Tabla 6. Especies de micobacterias ambientales aisladas en pacientes VIH/SIDA

\begin{tabular}{|lrr|}
\hline Especie & n & \multicolumn{1}{c|}{$\%$} \\
\hline M. tuberculosis & 20 & 76,9 \\
M. kansasii & 3 & 11,5 \\
MAI & 2 & 7,7 \\
M. chelonae & 1 & 3,8 \\
Total & 26 & 99,9 \\
\hline
\end{tabular}

cultivos falso-positivos, aunque cuando se encuentran en el lavado bronco-alveolar autores norteamericanos los consideran como causantes de micobacteriosis. Desgraciadamente los dos casos encontrados no fueron estudiados desde el punto de vista clínico.

De acuerdo a las normas existentes en nuestro país las muestras para identificación de micobacterias, cultivos de orina, sangre y ganglios deben derivarse al Laboratorio de Referencia para su diagnóstico; en cambio, cultivos de muestras pulmonares y extra-pulmonares, no requieren ser identificadas a este nivel si no aparentan anormalidad en la colonia. Sin embargo, dado que en aproximadamente un 9\% de los cultivos pueden aparecer MA, se debe ser muy estricto en derivar todas las cepas de aspecto anormal al Laboratorio de Referencia para su identificación real.

En relación a las especies encontradas por tipo de muestra, la gran mayoría corresponde a Complejo Mycobacterium tuberculosis y el resto a $M$. kansasii y M. avium, concordando con lo observado en estudios anteriores nacionales e internacionales $^{25-27}$. En sangre sigue siendo M. avium el más frecuente. Sin embargo, llama la atención que en muestras de pacientes VIH positivos sea $M$. kansasii y no M. avium el más frecuentemente 
Tabla 7. Antecedente de tratamiento antituberculoso del total de cepas estudiadas

\begin{tabular}{|c|c|c|c|c|}
\hline $\begin{array}{l}\text { Antecedentes } \\
\text { de tratamiento }\end{array}$ & $\mathbf{n}$ & Especies aisladas & $\mathbf{n}$ & $\%$ \\
\hline $\begin{array}{l}\text { Virgen a } \\
\text { tratamiento }\end{array}$ & $\begin{array}{r}397 \\
31\end{array}$ & $\begin{array}{l}\text { M. tuberculosis } \\
\text { MA* } \\
\text { M. kansasii } \\
\text { M. fortuitum } \\
\text { M. avium } \\
\text { M. peregrinum } \\
\text { M. chelonae } \\
\text { M. bovis BCG } \\
\text { M. gordonae } \\
\text { M. terrae } \\
\text { M. fortuitum-chelonae } \\
\text { M. No TBC crec rápid }\end{array}$ & $\begin{array}{r} \\
(9) \\
(5) \\
(3) \\
(3) \\
(3) \\
(3) \\
(2) \\
(1) \\
e \\
(1) \\
\text { do }\end{array}$ & $\begin{array}{r}92,2 \\
7,8 \\
\\
2,3 \\
1,3 \\
0,76 \\
0,76 \\
0,76 \\
0,76 \\
0,50 \\
0,25 \\
0,25 \\
0,25\end{array}$ \\
\hline $\begin{array}{l}\text { Control de } \\
\text { tratamiento }\end{array}$ & 75 & $\begin{array}{l}\text { M. tuberculosis } \\
\text { MA* } \\
\text { M. avium } \\
\text { M. kansasii }\end{array}$ & $\begin{array}{l}(68) \\
(4) \\
(3)\end{array}$ & $\begin{array}{r}90,1 \\
9,3 \\
\\
5,3 \\
4,0\end{array}$ \\
\hline A T: Recaída & 42 & $\begin{array}{l}\text { M. tuberculosis } \\
\text { MA* } \\
\text { M. kansasii } \\
\text { M. gordonae } \\
\text { M. terrae } \\
\text { M. abscessus }\end{array}$ & $\begin{array}{l}(37) \\
\\
\\
(2) \\
(1) \\
(1) \\
(1)\end{array}$ & $\begin{array}{r}88,0 \\
11,9 \\
\\
4,8 \\
2,4 \\
2,4 \\
2,4\end{array}$ \\
\hline $\begin{array}{l}\text { AT: Abandono } \\
\text { de tratamiento }\end{array}$ & 40 & $\begin{array}{l}\text { M. tuberculosis } \\
\text { MA* } \\
\text { M. chelonae } \\
\text { M. kansasii }\end{array}$ & $\begin{array}{l}\text { (35) } \\
\\
(3) \\
(2)\end{array}$ & $\begin{array}{r}87,5 \\
12,5 \\
7,5 \\
5,0\end{array}$ \\
\hline $\begin{array}{l}\text { Sin antecedentes } \\
\text { de tratamiento }\end{array}$ & 22 & $\begin{array}{l}\text { M. tuberculosis } \\
\text { MA* } \\
\text { M. gordonae } \\
\text { M. avium }\end{array}$ & $\begin{array}{l}(20) \\
\\
(1) \\
(1)\end{array}$ & $\begin{array}{r}90,9 \\
9,1 \\
\\
4,5 \\
4,5\end{array}$ \\
\hline $\begin{array}{l}\text { Anteriormente } \\
\text { tratado: NE }\end{array}$ & 9 & $\begin{array}{l}\text { M. tuberculosis } \\
\text { MA* } \\
\text { M. gordonae } \\
\text { M.avium }\end{array}$ & $\begin{array}{l}\text { (7) } \\
\\
(1) \\
(1)\end{array}$ & $\begin{array}{l}77,8 \\
22,2 \\
11,1 \\
11,1\end{array}$ \\
\hline Total & 585 & & & \\
\hline
\end{tabular}

*MA: micobacterias ambientales. AT: anteriormente tratado. NE no especificado.

aislado, como en otros estudios ${ }^{25,28}$. Pero, nuevamente, el número de aislamientos es muy escaso para llegar a una conclusión.

En nuestro país está normado el uso de las herramientas microbiológicas como baciloscopia, cultivo, pruebas de identificación y de susceptibilidad a drogas, todas las cuales se implementaron como resultado de estudios operativos realizados en nuestro laboratorio como una medida para optimizar el uso de estas técnicas. Es así como cultivos de pacientes vírgenes a tratamiento y con colonias de aspecto normal (rugosas, secas, acromógenas y eugónicas) no son sometidos a pruebas de identificación. En pacientes con antecedentes de tratamientos antituberculosos observamos una mayor proporción de aislamientos de MA, situación que puede deberse a que enfermos con historias de infecciones previas quedan más susceptibles a padecer colonizaciones o patologías francas por MA asentadas en lesiones pulmonares residuales. En caso de pacientes vírgenes a tratamiento, en una gran mayoría de ellos se aísla $M$. tuberculosis, y muchos de aquellos en los que crecen MA, corresponden a micobacterias saprófitas. Esto reafirma la estrategia de la norma de no proceder a la identificación de cultivos de pacientes vírgenes a tratamiento a no ser que se trate de colonias de aspecto anormal o pacientes VIH/SIDA.

Finalmente, en este estudio observamos que la frecuencia de MA ha aumentado de manera paulatina en el tiempo en nuestro medio, aunque manteniéndose prácticamente estable en esta última década, siendo el escenario de especies aisladas en general muy similar también a la observada en la década anterior. Además, reafirma las normas de derivación de cepas al Laboratorio de Referencia de Micobacterias para evitar errores diagnósticos que pueden llevar a prolongar tratamientos tan severos y largos como los de tuberculosis o micobacteriosis.

Desde un punto de vista clínico, se están encontrando cada vez más enfermos con micobacteriosis. Esta enfermedad puede presentarse en pacientes con inmunodepresiones sistémicas, como infectados con VIH/SIDA, o cuando existen condiciones que debilitan las defensas locales, afectando preferentemente los pulmones, ganglios linfáticos, piel y tejidos blandos ${ }^{2}$.

Las micobacteriosis de localización pulmonar pueden determinar formas cavitarias como la tuberculosis o presentarse en enfermos portadores de bronquiectasias, secuelas tuberculosas y fibrosis quística. Con menor frecuencia vemos micobacteriosis en EPOC avanzadas. Un grupo particular lo constituyen mujeres mayores, con un cuadro clínico caracterizado por bronquiectasias del 
Tabla 8. Frecuencia de micobacterias ambientales. Instituto de Salud Pública de Chile

\begin{tabular}{|c|c|c|c|c|}
\hline Año & $\begin{array}{c}\text { \% MA* } \\
\text { Muestras } \\
\text { pulmonares }\end{array}$ & $\begin{array}{c}\% \text { MA* } \\
\text { Muestras extra } \\
\text { pulmonares }\end{array}$ & $\begin{array}{c}\text { \% MA* } \\
\text { Muestras pulmonares y } \\
\text { extra pulmonares }\end{array}$ & $\begin{array}{c}\text { Incidencia de tuberculosis, } \\
\text { Chile (casos por } 100.000 \\
\text { habitantes) }\end{array}$ \\
\hline 1970 & 2,75 & - & - & - \\
\hline 1971-1972 & - & - & 11,4 & 86,2 \\
\hline 1975 & 2,31 & - & - & 81,3 \\
\hline 1977-1978 & - & $\begin{array}{c}5,6 \\
8,5 \text { sólo orinas }\end{array}$ & & 88,3 \\
\hline 1982 & 2,65 & - & - & 60,4 \\
\hline 1985 & 2,13 & - & - & 55,0 \\
\hline 1988-1989 & - & $\begin{array}{c}4,8 \\
6,1 \text { sólo orinas }\end{array}$ & - & 50,2 \\
\hline 1998-1999 & 5,7 & & & 29,2 \\
\hline 2008 & 8,3 & $\begin{array}{c}\text { 7,7 } \\
\text { 8,3 sólo orinas }\end{array}$ & 8,2 & 14,7 \\
\hline
\end{tabular}

*MA: Micobacterias ambientales.

lóbulo medio y de la língula, conocido como síndrome de Lady Windermere. En todos estos casos las MA más frecuentes son el complejo avium-intracelulare y $M$. kansassi.

La detección de una micobacteria ambiental en un cultivo no necesariamente significa enfermedad ya que puede ser solamente una colonización o, eventualmente, una contaminación. Para hacer el diagnóstico de micobacteriosis se requiere que haya por lo menos dos cultivos positivos con la misma MA en ausencia de M. tuberculosis, excepto en pacientes $\mathrm{VIH}+$, en muestras de lavado broncoalveolar o en la biopsia de un órgano; en estos casos, basta un solo aislamiento para presumir su rol patógeno.

El diagnóstico de micobacteriosis no implica la iniciación inmediata de un tratamiento. Nunca hay una urgencia por tratar y es mejor tomarse un tiempo antes de decidir la conducta a seguir. Esto fundamentalmente por tres razones: La terapia es prolongada, por lo menos un año y medio, y requiere del empleo de medicamentos no exentos de toxicidad; los resultados terapéuticos frecuentemente son pobres, y la evolución puede ser tan indolente que pareciera que muchos de estos pacientes sólo están colonizados. Por otra parte, los estudios de sensibilidad para MA no son tan confiables como los empleados para el $M$. tuberculosis y de hecho no son recomendados de rutina ${ }^{30}$.

Para decidirse a tratar un paciente portador de una micobacteriosis es necesario comprobar que existe progresión lesional radiológica, ya que los síntomas son más difíciles de evaluar, especial- mente en enfermos que tienen patologías de base, como bronquiectasias por ejemplo. Cuando se revisa la literatura, se aprecia que la mayoría de los pacientes en quienes se aislan MA, no reciben tratamiento.

Los esquemas terapéuticos recomendables son también muy heterogéneos debido a la frecuente y variada resistencia inicial de la mayoría de las MA a los fármacos antituberculosos habituales. En general las MA de crecimiento lento se tratan con asociaciones de rifampicina, etambutol y macrólidos (claritromicina o azitromicina), agregando un inyectable como estreptomicina, en los casos más avanzados, en tanto que las micobacteriosis de crecimiento rápido pueden curar con medicamentos habituales administrados por períodos más breves ${ }^{2}$.

Antes de decidir la terapia también es fundamental tener claro qué es lo que se pretende con el tratamiento. La erradicación de estas MA sólo se logra en menos de la mitad de los casos de acuerdo a las series más grandes publicadas. También es necesario considerar que muchos de estos enfermos, aunque curen pueden ser colonizados nuevamente con la misma MA u otra. Así por ejemplo, en una serie del British Medical Research Council a los cinco años de seguimiento sólo alrededor del 30\% de los pacientes estaban curados.

La decisión de iniciar una terapia antibiótica en un paciente con una micobacteria ambiental, es difícil. Frecuentemente es necesario observar por un tiempo al enfermo antes de tomar una decisión. 


\section{Agradecimientos}

Agradecemos a Ana María Jorquera, técnico de la Sección de Micobacterias del Instituto de Salud Pública de Chile, por su excelente apoyo técnico durante el estudio; a los encargados de los laboratorios de Tuberculosis de los hospitales de Arica, Iquique, Antofagasta, Coquimbo, Valparaíso, Linares, Chillán, Concepción, Talcahuano, Los Ángeles, Temuco, Puerto Montt, Coyhaique, Punta Arenas, CDT Eloísa Díaz, y de los hospitales de la Región Metropolitana Barros LucoTrudeau, Lucio Córdova, Sótero del Río, San Juan de Dios, San Borja-Arriarán y Luis Calvo Mackenna, por su compromiso y valiosa cooperación al enviar las cepas y antecedentes para el estudio. Sin ellos éste no hubiera sido posible. Al Dr. Victorino Farga, por su gran apoyo en la publicación de este trabajo.

\section{Bibliografía}

1.- DAWSON D J. Mycobacterial Terminology. J Clin Microbiol 2000, 38: 3913.

2.- FARGA V, CAMINERO J A. Tuberculosis. Editorial Mediterráneo, Tercera edición. Santiago, 2011. Págs. 449-69.

3.- PRIMM T P, LUCERO C A, FALKINHAM J O 3rd. Healths Impacts of Environmental Mycobacteria. Clin Microbiol Rev 2004; 17: 98-106.

4.- CRESPO ORTIZ M DEL P, CORRAL PRADO R, ALZATE A. Micobacterias no tuberculosas en personas VIH positivas y en personas sin factores de riesgo a la infección Colombia Médica 1997; 28: 136-44.

5.- VAN INGEN J, BOEREE M J, DEKHUIJZEN P N, VAN SOOLINGEN D. Environmental sources of rapid growing nontuberculosis mycobacteria causing disease in humans. Clin Microbiol Infect 2009; 15: 888-93.

6.- KANE A S, STINE C B, HUNGERFORD L, MATSCHE M, DRISCOLL C, BAYA A M. Mycobacterial as environmental portent in Chesapeake Bay fish species. Emerg Infect Dis 2007; 13: 329-31.

7.- $\quad$ SALAH IB, GHIGO E, DRANCOURT M. Free-living amoebae, a training field for macrophage resistance of mycobacteria. Clin Microbiol Infect 2009; 15: 894-905.

8.- LEÓN FRANCO C I. Presencia de las Micobacterias no tuberculosas en Colombia. Revista de los Estudiantes de Medicina de la Universidad Industrial de Santander. Médicas UIS 1998; 12: 181-7.

9.- GLASSROTH J. Pulmonary disease due to nontuberculous mycobacteria. Chest 2008; 133: 243-51.

10.- CAMPBELL I, DROBNIEWSKI F, NOVELLI V, ORMEROD P, POZNIAK A. Management of opportunist mycobacterial infections: Join Tuberculosis Committee guidelines 1999. Thorax 2000; 55: 210-18.

11.- KOECK J L, FABRE M, SIMON F, DAFFÉ M, GAR-
NOTEL E, MATAN A B, et al. Clinical characteristics of the smooth tubercle bacilli "Mycobacterium canettii" infection suggest the existence of an environmental reservoir. Clin Microbiol Infect 2011; 17: 1013-9.

12.- PIERSIMONI C, SCARPARO C. Extrapulmonary infectious associated with nontuberculous mycobacteria in inmunocompetent persons. Emerg Infect Dis 2009; 15: 1351-8.

13.- TORTOLI E. Clinical manifestations of nontuberculous mycobacteria infectious. Clin Microbiol Infect 2009; 15: 906-10.

14.- ALCAIDE F, ESTEBAN J. Cutaneous and soft skin infectious due to non-tuberculous mycobacteria. Enfermedades infecciosas y microbiología clínica 2010; 28 Suppl 1: 46-50.

15.- TORTOLI E, KROPPENSTEDT R, BARTOLONI A, CAROLI G, JAN I, PAWLOWSKY J, et al. S. Mycobacterium tusciae sp.nov. Int J Syst Bacteriol 1999, 49: 1839-44.

16.- CHERNILO S, TRUJILLO S, KAHN M, PAREDES M, ECHEVARRIA G, SEPÚLVEDA C. Enfermedades pulmonares en pacientes infectados con VIH hospitalizados en el Instituo Nacional del Tórax. Rev Med Chile 2005; 133: 517-24.

17.- PALOU DE COMASEMA P, RUIZ M, GUTIÉRREZ J. Descripción de un brote de infección cutánea por Mycobacterium abscessus tras masoterapia. http://fundacionio.org/docs/premio/casos/caso2_bacteriologia_2010. pdf

18.- GOBIERNO DE CHILE, MINSAL, Subsecretaría de Salud Pública. División de Prevención y Control de Enfermedades. Programa Nacional de Control de la Tuberculosis. Manual de Organización y Normas Técnicas. 2005.

19.- VELASCO M, VALENZUELA M T. Micobacterias no tuberculosas. Acta Médica FAB 1985; 8: 65-9.

20.- PONCE DE LEÓN L, LEPE R, VALENZUELA P, SALAS J. Análisis del estudio de cepas tipificadas en el Departamento de Tuberculosis en 1970. Bol Inst Bacteriológico Chile 1972; 14: 36-42.

21.- PONCE DE LEÓN L, LEPE R, PONCE J, SALDÍAS P, VALENZUELA P, FLORES M. Micobacterias atípicas. Evaluación de los estudios de tipificación en el Departamento de Tuberculosis en 1971-1972. Bol Inst Bacteriológico Chile 1973; 15: 17-20.

22.- PONCE DE LEÓN L, VELASCO M, BARRIOS J, LEPE R, PONCE J, VALENZUELA P. Frecuencia de Micobacterias Atípicas en pacientes investigados por sospecha de tuberculosis pulmonar. Bol Inst Bacteriológico Chile 1976; 18: 38-42.

23.- BARRIOS L, PONCE DE LEÓN L, VELASCO M, PONCE J, LEPE R, Valenzuela P Sensibilidad de las micobacterias atípicas a las drogas de acción antituberculosa. Bol Inst Salud Pública Chile 1979-1980; 21: 24-31.

24.- VELASCO M, VALENZUELA MT, LEPE R, PIFFAR- 
DI S, PONCE J. Frecuencia de Micobacterias no Tuberculosas en Chile. Rev Chil Infectol 1987; 4: 40-4.

25.- ARAYA P, VELASCO M, FERNÁNDEZ J. Identificación rápida de micobacterias no tuberculosas mediante análisis de patrones de restricción. Rev Med Chile 2006; 134: 868-73.

26.- MARTÍNEZ-MORAGÓN E, MENÉNDEZ R, PALASÍ P, SANTOS M, LÓPEZ J. Enfermedades por micobacterias ambientales en pacientes con y sin infección por el VIH: características epidemiológicas, clínicas y curso evolutivo. Arch Bronconeumol 2001; 37: 281-6.

27.- MODEROS L M, SIDKI FOFANA A, PEROVANI M A, GARCÍA G, MONTORO E H. Identificación de es- pecies micobacterianas en Cuba. Arch venez farmacol ter 2007; 26:142-5.

28.- BLANCARTE L, LUZ CAMPOS B, VILLANUEVA S S. Micobacterias atípicas en la República Mexicana. Salud pública Méx 1982; 24: 329-40.

29.- VELASCO M, PIFFARDI S, LEPE R, VALENZUELA P. Experiencia de una década en la tipificación y resistencia a fármacos antituberculosos en micobacterias aisladas de pacientes VIH/SIDA. Rev Chil Enf Respir 2000; 16:78-84.

30.- DALEY C L, GRIFFITH D E. Pulmonary non-tuberculous mycobacterial infection Intern J Tuberc Lung Dis 2010; 14: 665-71.

Correspondencia a:

Angélica Scappaticcio B.

Instituto de Salud Pública de Chile.

Avda. Marathon No 1000, Ñuñoa, Santiago.

E-mail: ascappaticcio@ispch.cl 